\title{
Oferta pública e privada de leitos e acesso aos cuidados à saúde na pandemia de Covid-19 no Brasil
}

\author{
Public and private supply of beds and access to health care in the \\ Covid-19 pandemic in Brazil
}

Danielle Conte Alves Riani Costa1, Ligia Bahia', Elza Maria Cristina Laurentino de Carvalho', Artur Monte Cardoso', Paulo Marcos Senra Souza'

DOI: $10.1590 / 0103-11042020$ E415

RESUMO A pandemia de Covid-19, uma crise sanitária global, pôs em xeque sistemas de saúde de diversos países. No Brasil, o atendimento aos pacientes trouxe à tona disparidades na oferta e no acesso a serviços públicos e privados, bem como iniciativas para preservar a segmentação assistencial. O trabalho sistematiza informações sobre: leitos para internação por Covid-19; pleitos de pacientes reivindicando acesso; e ações para ampliar a oferta de recursos assistenciais envolvendo proposições governamentais e setor privado (empresas de planos e hospitais). Houve expansão de leitos hospitalares, mas a distribuição desigual nas regiões do País não foi alterada; tampouco parece ter havido mudanças no padrão de controle de coberturas por parte das empresas de planos de saúde. Parcela significativa das ações judiciais analisadas refere-se à negação de acesso de clientes de planos privados por carência contratual, enquanto pacientes do Sistema Único de Saúde (SUS) pleitearam vaga em Unidade de Terapia Intensiva (UTI). Vidas foram perdidas em decorrência de omissões para proteção efetiva e qualificada. Unidades públicas de terapia intensiva tiveram ocupação máxima, enquanto o setor privado contabilizou leitos ociosos. A análise evidencia barreiras de acesso a leitos e resistência às tentativas de unificação de esforços públicos e privados para mitigar a letalidade pelo novo coronavírus.

PALAVRAS-CHAVE Infecções por coronavírus. Acesso aos serviços de saúde. Sistema Único de Saúde. Planos de pré-pagamento em saúde. Direito à saúde.

\begin{abstract}
The Covid-19 pandemic, a global health crisis, has put health systems in several countries in check. In Brazil, patient care has brought about disparities in the offer and access to public and private services and initiatives to preserve healthcare segmentation. The work systematizes information about: beds for hospitalization by Covid-19; patients' complaints claiming access; and actions to expand the supply of assistance resources involving government proposals and the private sector (health insurance companies and hospitals). There was an expansion of hospital beds, but the uneven distribution in the regions of the country has not changed, nor does it appear to be changes in the pattern of coverage control by health insurance companies. A significant portion of the analyzed law suits refers to the denial of access to private insurance clients due to contractual grace period, while Unified Health System (SUS) patients claimed a place in the Intensive Care Units (ICU). Lives were lost due to omissions for effective and qualified protection. Public Intensive Care Units had maximum occupancy, while the private sector accounted for empty beds. The analysis shows barriers to access to beds and resistance to attempts to unify public and private efforts to mitigate lethality by the new coronavirus.
\end{abstract}

1 Universidade Federal do Rio de Janeiro (UFRJ), Instituto de Estudos em Saúde Coletiva (lesc) - Rio de Janeiro (RJ), Brasil. danielle.conte@gmail.com
KEYWORDS Coronavirus infections. Health services accessibility. Unified Health System. Prepaid health plans. Right to health. 


\section{Introdução}

O Sistema Único de Saúde (SUS) ocupou, ao lado do monitoramento da disseminação de casos e mortes por Covid-19, o centro do debate público durante a pandemia. No que diz respeito à resposta, o SUS foi testado em relação ao planejamento, organização, financiamento e prestação da assistência. O Brasil apresenta flagrantes desigualdades geográficas, desproporção de recursos para acesso e uso segundo estratificação e tipo de procedimento. Os leitos de internação de maior complexidade no País, como os de Unidade de Terapia Intensiva (UTI) Tipo III, têm uma tendência de concentração ainda maior nas regiões Sul (2 leitos/100 mil habitantes) e Sudeste (1,5 leitos/100 mil habitantes). Já na região Norte, a taxa é de 0,03 leitos de UTI Tipo III/100 mil habitantes, agravada pelo fato de mais de $90 \%$ desse tipo de leito estar disponível somente para o setor privado ${ }^{1}$. Dessa forma, os recursos assistenciais em saúde tendem a ser triplamente concentrados de acordo com características regionais, demanda e oferta pública ou privada, e complexidade dos recursos assistenciais.

Após anos de subfinanciamento, o SUS se vê diante de um dos maiores desafios desde a sua criação. Expandir leitos, adquirir equipamentos e insumos passou a ser prioridade. Lidar com as desigualdades regionais na prestação da assistência à saúde passou a ser uma realidade. Articular uma estratégia público-privada para atender a todos os acometidos tornou-se uma possibilidade. O sistema universal, ainda que com bases financeiras frágeis, evidenciou suas potencialidades técnicas e políticas.

A principal estratégia que os países que nos antecederam no enfrentamento da Covid-19 encontraram para diminuir a disseminação foi o distanciamento social ${ }^{2}$ e, após o controle e diminuição constante do número de casos, o retorno gradual e programado de determinadas atividades econômicas ${ }^{\mathbf{3} 4}$.

No Brasil, a doença se alastrou a partir das capitais. No mês de junho de 2020, deu-se início à flexibilização do isolamento social em algumas localidades sem que os requisitos preconizados pela Organização Mundial da Saúde (OMS) para suspensão gradativa das medidas de isolamento tenham sido observados ${ }^{4,5}$. O acesso a leitos está sendo utilizado como principal parâmetro para flexibilizar o isolamento social6,7, de modo que, se houver leito disponível, a flexibilização é permitida independentemente de critérios epidemiológicos.

Considerando a necessidade de o sistema de saúde viabilizar atendimento célere e oportuno durante a pandemia, busca-se levantar informações que permitam uma aproximação às condições de acesso aos serviços públicos e privados. Destarte, o objetivo do presente trabalho foi buscar evidências sobre a ocorrência de mudanças na oferta e acesso a serviços de saúde públicos e privados no Brasil durante a pandemia de Covid-19, identificando aspectos que nos aproximam ou nos afastam do princípio de universalidade do SUS.

\section{Material e métodos}

Trata-se de um estudo descritivo, de natureza exploratória, no qual se esquadrinham problemas para acesso aos serviços públicos e privados de saúde durante a pandemia no Brasil, tendo como base três dimensões: oferta de leitos para internação por Covid-19; pleitos reivindicando acesso a serviços; e iniciativas para uso de recursos assistenciais privados pelo SUS. Esse delineamento metodológico pode ser observado na matriz do estudo (figura 1). Para tanto, foram consultados dados secundários disponíveis em fontes governamentais de órgãos dos poderes Executivo, Legislativo e Judiciário; bem como pesquisa bibliográfica, documental e de notícias veiculadas pela mídia especializada e comercial.

$\mathrm{O}$ acesso a leitos foi avaliado por meio da quantidade de leitos de Unidades de Terapia Intensiva (UTI) públicos e privados direcionados para tratamento de Covid-19, além de estimativas sobre o acesso da população que 
recorreu aos serviços públicos e privados de saúde, englobando taxa de ocupação, ocorrência de filas e dificuldade para atendimento.

Foi pesquisada a quantidade de novos leitos direcionados, exclusivamente, para tratamento de Covid-19 no Cadastro Nacional de Estabelecimentos de Saúde (CNES), disponível no Datasus e obtidos via Tabnet ${ }^{1}$, quais sejam os leitos complementares 'UTI II Adulto Covid-19' e 'UTI II Pediátrica Covid-19', incluídos na tabela de leitos do CNES mediante regulamentação específica ${ }^{\mathbf{8}, \mathbf{9}}$.

Os leitos complementares são categorizados no CNES, conforme sua habilitação, como 'SUS' ou 'não SUS'. O leito complementar 'SUS' corresponde àquele habilitado pelo Ministério da Saúde (MS) em portaria. O leito 'não SUS' pode ter dois significados: leito privado não utilizado no SUS ou leito utilizado no SUS (em hospital público ou privado), mas ainda não habilitado pelo $\mathrm{MS}^{10,11}$.

Os leitos complementares de UTI II Covid19 categorizados no CNES como 'não SUS', mas localizados em estabelecimentos públicos de saúde, foram identificados neste estudo como leitos 'SUS não habilitados', visto que prestam atendimento no SUS, mas ainda não constam em portaria do MS. Os leitos categorizados no CNES como 'não SUS' e situados em estabelecimentos privados foram identificados como 'não SUS não habilitados'.

A análise dos pleitos reivindicando acesso a serviços públicos e privados de saúde baseou-se na consulta a nas decisões judiciais e ao registro de reclamações na Agência Nacional de Saúde Suplementar (ANS) ${ }^{\mathbf{1 2}}$. As ações judiciais foram consultadas em boletins divulgados no sítio eletrônico dos Tribunais de Justiça dos estados do Rio de Janeiro e de São Paulo, os quais consolidaram um repositório de decisões de tribunais de justiça de todo o País e do Supremo Tribunal Federal (STF) que tiveram como escopo a pandemia de Covid-1913-15. Foram consultadas as decisões relacionadas com o acesso a serviços de saúde informadas nos boletins divulgados pelos referidos tribunais entre $1^{\circ}$ de março e 13 de julho de 2020. Os dados sobre reclamações efetuadas por consumidores de planos de saúde, no período de $1^{\circ}$ de março a 26 de junho de 2020, foram obtidos a partir de informações disponibilizadas pelo órgão regulador ${ }^{12}$.

Por fim, as relações entre o SUS e o setor privado assistencial, considerando planos de saúde e hospitais privados, foram analisadas a partir das ações realizadas e medidas propostas para oportunizar o acesso, por meio de documentos que permitiram identificar o posicionamento de órgãos públicos, da ANS e de entidades empresariais.

Entre as principais limitações do estudo, situa-se o possível hiato de tempo no registro e na habilitação de leitos complementares 'SUS' e 'não SUS'. Leitos Covid-19 categorizados como 'não SUS não habilitados' podem pertencer a estabelecimentos privados que prestam atendimento ao SUS, mas ainda não foram habilitados em portaria.

Não é possível afirmar que os dados abarcam todos os leitos Covid-19, em especial quanto aos leitos localizados em estabelecimentos privados que prestam atendimento a planos privados de saúde, pois não necessariamente atualizaram a base de dados do CNES.

No que concerne às decisões judiciais, as ações compiladas pelos Tribunais de Justiça do Rio de Janeiro e de São Paulo incluem decisões de outras unidades federativas, mas podem não alcançar a totalidade de casos judicializados no País durante a pandemia. 
Figura 1. Matriz metodológica do estudo

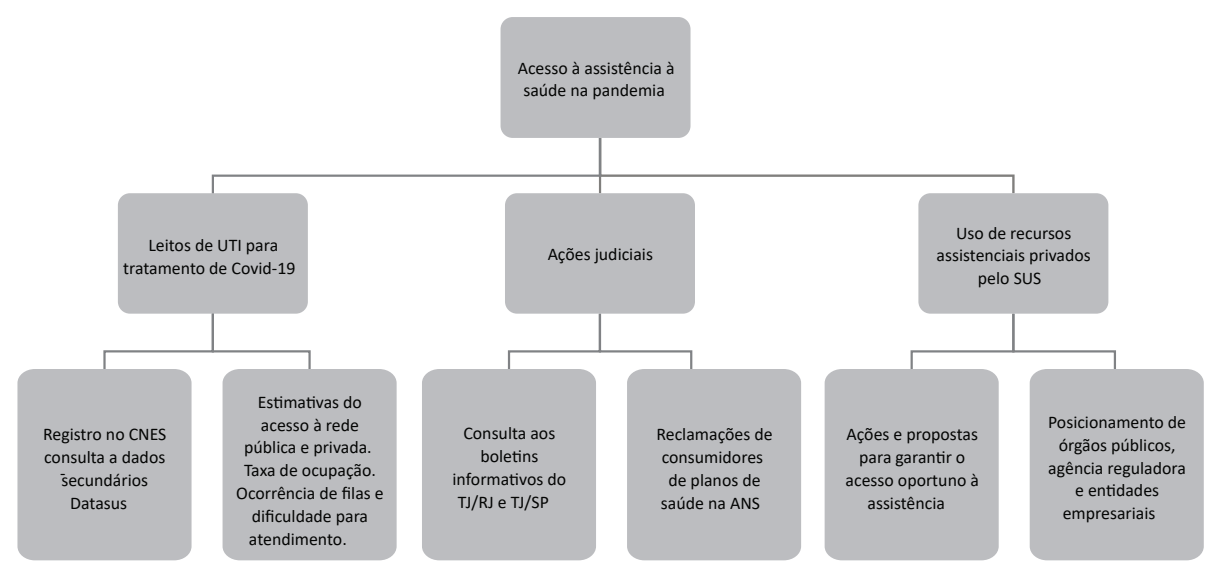

Fonte: Elaboração própria.

\section{Resultados e discussão}

\section{O SUS e o setor privado na pandemia: oferta e acesso a leitos para tratamento da Covid-19}

A despeito da declaração de emergência de saúde pública pela $\mathrm{OMS}^{16}$ diante da gravidade evidenciada no cenário internacional pelo aumento exponencial de casos em diversos países e do primeiro caso confirmado de Covid-19 no Brasil ter ocorrido em fevereiro de $2020^{17}$, somente em março foi publicada pelo MS a portaria que autorizou a habilitação de leitos de UTI Adulto e Pediátrico para atendimento exclusivo dos pacientes com Covid-19, com custeio diferenciado para diária hospitalar ${ }^{8}$. O processo de habilitação desses leitos no SUS somente teve início em abril, momento em que o País passou a registrar mais de 85 mil casos, o número de óbitos saltou de 242 para quase 6 mil17 e alguns municípios registravam taxa de ocupação de leitos UTI acima de 90\% ${ }^{18-21}$. Provavelmente, esses leitos já estavam disponíveis para o SUS.

No âmbito nacional, os primeiros 2.409 'leitos SUS' foram habilitados em abril de 2020 e aumentaram para 8.764 em junho. Somada aos 'leitos SUS não habilitados', no período de março a junho, a oferta da rede SUS triplicou, totalizando 15.662 leitos, o que decorreu de esforços no âmbito público (aumento de leitos em estabelecimentos públicos e hospitais de campanha).

A cidade de São Paulo apresentou maior incremento de 'leitos SUS', habilitados ou não, totalizando mais de 3.700 leitos Covid-19 em junho. Outros estados cujas capitais enfrentaram colapso do sistema, como Ceará, Pará e Rio de Janeiro ${ }^{22,23}$, também tiveram aumento expressivo do número de leitos SUS (tabela 1). 
Tabela 1. Número de leitos de UTI* 'SUS habilitados', 'SUS não habilitados' e 'não SUS' para tratamento da Covid-19, por Unidade Federativa (UF) e regiões do País, no período de março a junho de 2020. Brasil

Quantidade SUS não habilitados

Quantidade não SUS (estabelecimento

Quantidade SUS habilitados ${ }^{\star \star}$

(administração pública)

privado)

\begin{tabular}{|c|c|c|c|c|c|c|c|c|c|c|c|c|c|c|}
\hline \multirow[b]{2}{*}{ UF } & \multicolumn{5}{|c|}{ Quantidade SUS habilitados*^ } & \multicolumn{5}{|c|}{ (administração pública) } & \multirow[b]{2}{*}{$2020 /$} & \multirow[b]{2}{*}{ 2020/ } & \multirow[b]{2}{*}{$\begin{array}{r}2020 / \\
\text { Jun }\end{array}$} & \multirow{2}{*}{$\begin{array}{r}\text { privado) } \\
\text { Variação } \\
\text { maio e } \\
\text { junho }\end{array}$} \\
\hline & $\begin{array}{r}2020 / \\
\text { Abr }\end{array}$ & $\begin{array}{r}2020 / \\
\text { Maio }\end{array}$ & $\begin{array}{r}2020 / \\
\text { Jun } \\
\end{array}$ & $\begin{array}{r}\text { Variação } \\
\text { maio e } \\
\text { junho } \\
\end{array}$ & $\begin{array}{r}2020 / \\
\text { Mar }\end{array}$ & $\begin{array}{r}2020 / \\
\text { Abr }\end{array}$ & $\begin{array}{r}2020 / \\
\text { Maio }\end{array}$ & $\begin{array}{r}2020 / \\
\text { Jun }\end{array}$ & $\begin{array}{r}\text { Variação } \\
\text { maio e } \\
\text { junho } \\
\end{array}$ & $\begin{array}{r}2020 / \\
\text { Mar }\end{array}$ & & & & \\
\hline Rondônia & 38 & 45 & 81 & 36 & 10 & 25 & 46 & 57 & 11 & 0 & 0 & 22 & 21 & -1 \\
\hline Acre & 10 & 10 & 30 & 20 & 10 & 44 & 44 & 34 & -10 & 0 & 0 & 0 & 0 & 0 \\
\hline Amazonas & 144 & 194 & 194 & 0 & 4 & 109 & 87 & 59 & -28 & 0 & 0 & 0 & 0 & 0 \\
\hline Roraima & 0 & 0 & 15 & 15 & 0 & 20 & 30 & 20 & -10 & 0 & 0 & 0 & 0 & 0 \\
\hline Pará & 32 & 300 & 316 & 16 & 10 & 242 & 286 & 413 & 127 & 2 & 12 & 6 & 6 & 0 \\
\hline Amapá & 0 & 32 & 32 & 0 & 13 & 33 & 11 & 11 & 0 & 0 & 0 & 0 & 0 & 0 \\
\hline Tocantins & 0 & 42 & 42 & 0 & 0 & 26 & 0 & 17 & 17 & 0 & 14 & 5 & 5 & 0 \\
\hline Norte & 224 & 623 & 710 & 87 & 47 & 499 & 504 & 611 & 107 & 2 & 26 & 33 & 32 & -1 \\
\hline Maranhão & 110 & 212 & 228 & 16 & 31 & 94 & 134 & 157 & 23 & 30 & 38 & 28 & 8 & -20 \\
\hline Piauí & 20 & 92 & 138 & 46 & 198 & 160 & 150 & 168 & 18 & 25 & 42 & 33 & 43 & 10 \\
\hline Ceará & 200 & 362 & 382 & 20 & 333 & 511 & 314 & 371 & 57 & 20 & 123 & 126 & 101 & -25 \\
\hline $\begin{array}{l}\text { Rio Grande do } \\
\text { Norte }\end{array}$ & 10 & 140 & 142 & 2 & 160 & 248 & 204 & 222 & 18 & 0 & 65 & 65 & 70 & 5 \\
\hline Paraíba & 0 & 105 & 170 & 65 & 0 & 83 & 61 & 71 & 10 & 4 & 5 & 6 & 15 & 9 \\
\hline Pernambuco & 228 & 408 & 634 & 226 & 514 & 586 & 594 & 509 & -85 & 68 & 136 & 207 & 126 & -81 \\
\hline Alagoas & 132 & 154 & 154 & 0 & 67 & 53 & 84 & 91 & 7 & 55 & 20 & 31 & 31 & 0 \\
\hline Sergipe & 44 & 54 & 54 & 0 & 0 & 1 & 10 & 16 & 6 & 24 & 0 & 48 & 90 & 42 \\
\hline Bahia & 90 & 188 & 529 & 341 & 517 & 777 & 638 & 530 & -108 & 166 & 176 & 182 & 146 & -36 \\
\hline Nordeste & 834 & 17.15 & 2.431 & 716 & 1.820 & 2.513 & 2.189 & 2.135 & -54 & 392 & 605 & 726 & 630 & -96 \\
\hline Minas Gerais & 40 & 95 & 418 & 323 & 96 & 558 & 477 & 531 & 54 & 94 & 898 & 971 & 953 & -18 \\
\hline Espírito Santo & 88 & 165 & 165 & 0 & 47 & 249 & 207 & 260 & 53 & 10 & 123 & 161 & 186 & 25 \\
\hline Rio de Janeiro & 40 & 510 & 733 & 223 & 878 & 1.423 & 1.155 & 1.250 & 95 & 87 & 219 & 207 & 181 & -26 \\
\hline São Paulo & 734 & 2.001 & 2.056 & 55 & 1.281 & 1.420 & 1.544 & 1.647 & 103 & 445 & 997 & 963 & 1.430 & 467 \\
\hline Sudeste & 902 & 2.771 & 3.372 & 601 & 2.302 & 3.650 & 3.383 & 3.688 & 305 & 636 & 2.237 & 2.302 & 2.750 & 448 \\
\hline Paraná & 30 & 435 & 473 & 38 & 353 & 331 & 30 & 158 & 128 & 467 & 281 & 82 & 181 & 99 \\
\hline $\begin{array}{l}\text { Santa Cata- } \\
\text { rina }\end{array}$ & 180 & 372 & 453 & 81 & 79 & 95 & 51 & 34 & -17 & 113 & 181 & 146 & 114 & -32 \\
\hline $\begin{array}{l}\text { Rio Grande } \\
\text { do Sul }\end{array}$ & 30 & 275 & 586 & 311 & 30 & 91 & 76 & 35 & -41 & 381 & 424 & 257 & 173 & -84 \\
\hline Sul & 240 & 1082 & 1512 & 430 & 462 & 517 & 157 & 227 & 70 & 961 & 886 & 485 & 468 & -17 \\
\hline $\begin{array}{l}\text { Mato Grosso } \\
\text { do Sul }\end{array}$ & 25 & 139 & 157 & 18 & 4 & 33 & 23 & 5 & -18 & 10 & 32 & 31 & 34 & 3 \\
\hline Mato Grosso & 0 & 198 & 258 & 60 & 251 & 269 & 80 & 20 & -60 & 18 & 15 & 8 & 40 & 32 \\
\hline Goiás & 104 & 139 & 139 & 0 & 94 & 172 & 133 & 192 & 59 & 12 & 13 & 37 & 129 & 92 \\
\hline $\begin{array}{l}\text { Distrito Fe- } \\
\text { deral }\end{array}$ & 80 & 185 & 185 & 0 & 0 & 0 & 20 & 20 & 0 & 0 & 0 & 0 & 80 & 80 \\
\hline Centro-Oeste & 209 & 661 & 739 & 78 & 349 & 474 & 256 & 237 & -19 & 40 & 60 & 76 & 283 & 207 \\
\hline Total & 2.409 & 6.852 & 8.764 & 1.912 & 4.980 & 7.653 & 6.489 & 6.898 & 409 & 2.031 & 3.814 & 3.622 & 4.163 & 541 \\
\hline
\end{tabular}

Fonte: Brasil. Ministério da Saúde. Datasus. março a junho 2020 1.

* UTI adulto II Covid-19 e UTI pediátrica II Covid-19.

** Em março de 2020, não havia registro de leitos UTI adulto II Covid-19 e UTI pediátrica II Covid-19 habilitados pelo Ministério da Saúde. 
A despeito da ampliação da capacidade instalada, nota-se que a abertura de leitos manteve desigualdades existentes, com incremento maior na região Sudeste e menor na região Norte (figura 2).

Figura 2. Quantidade de leitos de UTI para tratamento de Covid-19 por 100 mil habitantes, em maio de 2020, por regiões do País. Brasil

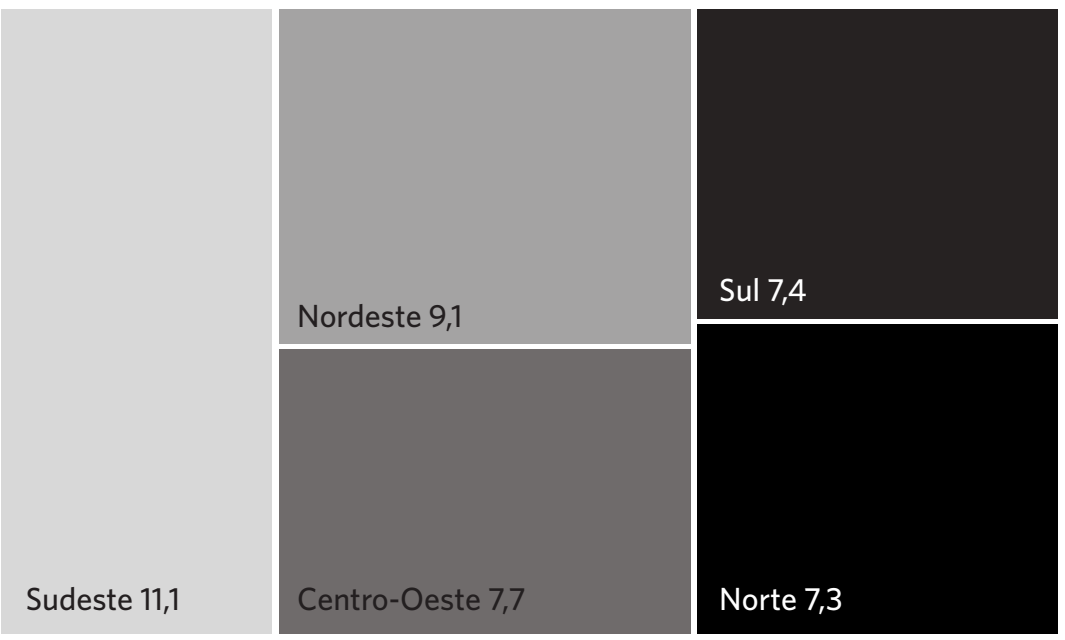

Fonte: Elaboração própria, com base em dados do Ministério da Saúde. Datasus (número de leitos) e Painel Coronavírus (número de habitantes) ${ }^{17} .2020$.

Cabe registrar a importância do CNES como uma fonte para análises de leitos e recursos disponíveis nos estabelecimentos de saúde do País e que a habilitação dos leitos Covid-19 possibilita o acesso a recursos diferenciados no âmbito do SUS. Entretanto, a referida base não possibilita, ao menos no presente momento, análises mais robustas sobre o quantitativo de leitos privados Covid-19, não permitindo conhecer com precisão a capacidade instalada no País, indicando uma vulnerabilidade do sistema e abrindo brecha para interpretações equivocadas a respeito dos recursos assistenciais disponíveis.

A ANS requisitou informação para 109 empresas de planos de saúde que possuíam hospitais próprios. As informações provenientes de 50 empresas respondentes indicaram taxas de ocupação de leitos alocados para atendimento à Covid-19 de $45 \%$ e $61 \%$ em abril e maio respectivamente ${ }^{24}$.
Enquanto isso, o SUS registrava fila de espera para internação e taxas de ocupação de leitos próximo da capacidade máxima em diversas localidades do País ${ }^{22,23}$. Ainda em maio, hospitais privados iniciaram a redução da quantidade de leitos para atendimento a esses $\operatorname{casos}^{25}$. Fica evidente, portanto, a desigualdade no acesso aos serviços públicos e privados de saúde, principalmente, pela desproporção entre potencial demanda privada e oferta, visto que a população vinculada a planos de saúde representa cerca de $24 \%$ da população ${ }^{26}$.

Seguindo a tendência do setor privado, em junho, foi iniciado o fechamento de leitos públicos e hospitais de campanha em algumas cidades ante a redução da taxa de ocupação de leitos $\mathbf{2 7 , 2 8}$.

Os resultados do presente estudo estão em consonância com achados da literatura que demonstram discrepâncias na distribuição 
de leitos e recursos no País, repercutindo na capacidade de resposta à doença ${ }^{29,30}$.

Em que pese a importância do SUS no enfrentamento da pandemia, pode-se afirmar que a implementação de leitos públicos ocorreu tardiamente e não foi suficiente para garantir o pleno atendimento e o direito à saúde em diferentes cidades. O cenário configurado por fila de espera no SUS versus leitos ociosos e encerrados no setor privado, em um contexto de pandemia em curso, preservou e acirrou desigualdades de acesso.

Assim como em outros países, hospitais foram reorganizados, com parte dos recursos físicos e humanos anteriormente alocados aos procedimentos de diferentes especialidades médicas sendo adaptados para cuidados intensivos. No entanto, ainda é incerto o quanto as modificações serão absorvidas e qual será o real legado para os sistemas de saúde ${ }^{31}$.

\section{Ações judiciais e reclamações envolvendo a garantia de acesso aos serviços de saúde}

No período de $1^{\circ}$ de março a 13 de julho de 2020, foram identificadas 23 decisões judiciais envolvendo a garantia de acesso durante a pandemia. A maior parte das ações foi proveniente de planos de saúde, tal como vem sendo assinalado pela literatura ${ }^{32}$. Das 14 ações judiciais voltadas para o acesso a planos privados de saúde, 8 estavam relacionadas com a cobertura independentemente do prazo de carência; e nenhuma, com a dificuldade de leito disponível para internação. Entre as nove ações relacionadas ao SUS, três estabeleceram prazos para a instalação de novos leitos e hospitais de campanha, e quatro delas pleitearam internações.

Grande parte das decisões foi favorável aos cidadãos, tal como apontado em outros estudos 33-35. Embora a via judicial não tenha sido muito acionada, fica evidente que, enquanto no setor público os usuários buscaram acesso à internação em UTI por falta de vaga, não foi identificada ação judicial de consumidores de planos de saúde pleiteando vaga de terapia intensiva por falta de leitos, mas, sim, acesso para clientes que estavam cumprindo o prazo de carência contratual.

A dificuldade de acesso à UTI por pacientes do SUS precede a pandemia e é exacerbada por esta, configurando, o judiciário, importante espaço para preservação de direitos. A busca de acesso pela via judicial reitera a busca de direitos individuais, mesmo quando as estratégias de enfrentamento recomendadas são coletivas.

No que se refere às reclamações efetuadas pelos clientes de planos privados na ANS, entre $1^{\circ}$ de março e 26 de junho de 2020 , foram contabilizados o total de 5.333 queixas relacionadas com o tema coronavírus, sendo 49 delas por 'ausência de rede para tratamento ou internação'12.

\section{Relações entre o SUS e o setor privado hospitalar e empresas de planos de saúde na pandemia}

É possível destacar alguns fatores relativos à falta de acesso a internações no SUS: demora para se organizar para enfrentamento da pandemia; leitos de hospitais de campanha que não saíram do papel; atraso na liberação de recursos financeiros; e o fator mais decisivo que poderia ter mudado completamente o panorama da pandemia no Brasil: a falta de integração para regulação de leitos públicos e privados e fila única para atendimento a casos graves de Covid-19.

A proposta para unificar os leitos de internação e de UTI públicos e privados para o enfrentamento da pandemia, lançada pelas campanhas unificadas 'Leitos para todos' e 'Vidas iguais ${ }^{\mathbf{3 6}, \mathbf{3 7}}$, consistiu na criação de um sistema nacional de vagas que seria regulamentado pelo MS em articulação com estados, municípios e prestadores de serviços, e na regulação de centrais únicas de vagas pelas secretarias de saúde estaduais ${ }^{38-40}$.

Tal medida, que embasou o Projeto de Lei ${ }^{\circ}$ $2.324 / 2020^{41}$, aprovado pelo Senado Federal, está legalmente prevista na Constituição Federal, na Lei Orgânica da Saúde e na Lei 
$\mathrm{n}^{\mathrm{0}} 13.979 / 2020^{\mathbf{4 2}}$, sobre medidas para enfrentamento da situação de emergência. Contudo, a requisição de leitos privados não foi a opção adotada pelo governo federal mesmo na fase mais crítica da doença, na qual capitais apresentaram filas de espera de pacientes para leitos públicos.

Consultada pelo MS a respeito da recomendação do Conselho Nacional de Saúde (CNS), que propõe a requisição de leitos privados pelo SUS e a regulação única durante a pandemia ${ }^{43}$, a Diretoria Colegiada da ANS manifestou, em documento aprovado por quatro dos cinco diretores, que a gestão unificada de leitos poderia "gerar o sentimento de restrição de acesso pela ótica do beneficiário"44(6) de planos privados de saúde e "levar à desarticulação da rede assistencial privada”44(7). Além disso, a

gestão unificada dos leitos públicos e privados para o enfrentamento da Covid-19 seria fator de forte impacto sobre organização do acesso dos beneficiários à rede hospitalar para outras necessidades em saúde44(5).

O referido documento menciona, ainda, que a "hipótese de gestão unificada dos leitos públicos e privados [...] dependeria de uma regulação que equilibrasse oferta e demanda [...] de modo a ocupar leitos efetivamente ociosos"44(5) e que, predominantemente, a precificação das internações no setor suplementar de saúde é negociada caso a caso, de acordo com a unidade hospitalar.

Em resposta à recomendação do CNS, entidades representativas de hospitais e planos privados se manifestaram contrariamente. A Confederação Nacional de Saúde (CNSaúde) alegou desorganização da rede privada, possibilidade de disputa judicial por leitos e descrédito dos planos de saúde ${ }^{45}$.

As deficiências do SUS e a ausência de sistema de gestão para conduzir a fila única foram as justificativas apresentadas pela Federação Nacional dos Corretores de Seguros Privados (Fenacor) e pela Federação Nacional de Saúde Suplementar (FenaSaúde) ${ }^{\mathbf{4 6}, 47}$. A
Fenacor acrescenta que a baixa ocupação na rede privada ocorre por reserva de leitos para atender a outras enfermidades e propõe como alternativa que o SUS continue adquirindo leitos nos hospitais privados.

Destoando do coro dos contrários ao uso de leitos privados durante a pandemia, a Federação Brasileira de Hospitais (FBH) estimou que $80 \%$ dos hospitais privados do País estavam ociosos e sugeriu a contratação desses leitos que estariam prontos para receber pacientes do SUS ${ }^{45}$, o que apoiaria hospitais de pequeno e médio impactados pela queda de receita ${ }^{\mathbf{8}}$.

A Associação Nacional dos Hospitais Privados (Anahp) ratifica a queda da receita dos hospitais, porém, posiciona-se contrária à unificação dos leitos, argumentando ausência de planejamento, desestruturação do setor suplementar, insegurança jurídica e deficiências estruturais do sistema de saúde ${ }^{49}$.

Os valores oferecidos pela diária de UTI do MS também foram considerados insatisfatórios, embora a portaria que trata da habilitação de leitos de UTI para tratamento de Covid-19 tenha sido republicada para dobrar o valor da diária para $\mathrm{R} \$ 1.600,00^{8}$.

A fim de evitar a fila única e a requisição administrativa de leitos pelo setor público sem remuneração acordada, associações representativas de hospitais privados e de empresas de planos de saúde apresentaram quatro propostas ao MS: revitalização de leitos públicos fechados, construção de hospitais de campanha, testagem da população e abertura de editais públicos para contratação de leitos e serviços ${ }^{50}$.

Argumentos contrários aos dos setores empresariais foram elencados por pesquisadores e entidades representativas de movimentos sociais. A unificação dos leitos públicos e privados, medida calcada no arcabouço jurídico, prevê a utilização de leitos privados por indivíduos que não possuem plano de saúde mediante indenização $0^{51}$, cabendo destacar que a contratação de estabelecimentos privados e filantrópicos para atendimento a pacientes 
do SUS é uma prática já adotada no Brasil. Ademais, deixar de viabilizar o atendimento oportuno e efetivo a usuários que estão na fila do SUS sob a justificativa de que pode faltar leito para consumidores de planos de saúde, que não apresentam problemas de acesso, não parece plausível.

Em que pese o posicionamento de órgãos e entidades representativas, a unificação dos leitos deveria ser uma decisão do governo federal em pactuação com os atores envolvidos. Estabelecer acordos sobre valores de remuneração e pormenores procedimentais e viabilizar a adoção dessa medida mitigando discordâncias são tarefas para autoridade sanitária nacional.

$\mathrm{O}$ avanço avassalador de uma pandemia exige celeridade no planejamento. A gestão unificada de leitos não pretende sanar as deficiências do sistema de saúde, e, sim, garantir atendimento igualitário a pessoas com diagnóstico ou suspeita de Covid-19. Experiências da Austrália, da Espanha, da Irlanda e do Reino Unido, países que uniram hospitais públicos e privados para atendimento durante a pandemia, demonstram a factibilidade da proposta ${ }^{38,39}$. Inclusive, é importante ressaltar que os recursos orçamentários para enfrentamento da pandemia poderiam ter sido utilizados para organizar uma rede assistencial efetiva, entretanto, apenas uma parcela do orçamento autorizado foi empenhada e executada pelo governo federal52

O Conselho Nacional de Justiça (CNJ) se manifestou pela adoção de medidas de gestão voltadas à prevenção da judicialização da saúde durante a pandemia, recomendando o diálogo entre as esferas pública e privada e a centralização da gestão da rede de serviços regionais/ estaduais. No detalhamento das proposições, sugeriu a contratação dos leitos privados a serviço do SUS por meio de chamamentos públicos e mencionou a possibilidade de requisição de recursos hospitalares privados ${ }^{53}$.

Algumas iniciativas caminharam nessa direção, com a publicação de editais de chamamento público para hospitais privados no Rio Grande do Sul ${ }^{54}$, bem como a publicação de decretos prevendo a possibilidade de requisição de leitos particulares em São Paulo/SP55, Nova Friburgo/RJ56 e Maranhão ${ }^{57}$, por exemplo. O governo do Distrito Federal, por sua vez, publicou decreto requisitando 65 leitos de UTI, insumos e profissionais de saúde de hospitais particulares, mas o normativo foi revogado após acordo para que hospitais privados cedessem 35 leitos de UTI para a rede pública ${ }^{58}$.

À exceção de algumas experiências isoladas, a atuação do setor privado se restringiu a doações de grandes empresas para a rede pública, podendo-se citar: Grupo Fleury, Rede Ímpar, Rede D'Or, Droga Raia, Drogasil, Eurofarma Qualicorp e UnitedHealth Group Brasil, assim como realizado por estatais, empresas privadas, empresas do setor bancário e pessoas físicas ${ }^{52,59}$. Sob a justificativa de que a situação de emergência poderia se agravar no País e que, assim, faltariam leitos para os consumidores de planos de saúde, a letargia e as lacunas de coordenação das respostas do governo brasileiro foram funcionais à manutenção da segmentação: cada setor atende a uma determinada parcela da população.

Dessa forma, a crise sanitária gerada pela Covid-19 agrava necessidades de saúde existentes e expõe fragilidades de um sistema de saúde subfinanciado e depreciado ao longo dos anos, com toda a sua potência relegada ao status de 'SUS para pobres', enquanto o setor privado de saúde tenta fortalecer uma imagem positiva com doações ao setor público.

Apesar de as contribuições da rede hospitalar privada terem sido importantes em um contexto de desprovimento de recursos e demora para organizar a rede hospitalar pública, constatou-se que o potencial de sinergia na interação entre SUS e planos e hospitais privados para salvar vidas foi reduzido a mero auxílio para atenuar lacunas pontuais do SUS.

\section{Considerações finais}

A desigualdade no acesso aos serviços públicos e privados de saúde permaneceu latente 
durante a pandemia de Covid-19. Expansão de leitos públicos e perfis de mortalidade diferenciados por região do País, demora para abertura de novos leitos no SUS, ausência de informações sobre a capacidade instalada em hospitais privados, falta de interação entre setores público e privado para mitigar a letalidade pelo novo coronavírus; e pacientes do SUS recorrendo ao Judiciário por vaga em UTI, enquanto os consumidores de planos de saúde pleitearam a suspensão dos prazos de carência contratual, marcaram os principais achados do presente estudo.

Cabe acompanhar qual será o legado da expansão do número de leitos e de recursos para o SUS, não obstante parte dessa expansão seja fugaz, uma vez que hospitais de campanha estejam sendo desmontados.

A expansão de leitos de terapia intensiva não reduziu as discrepâncias entre as regiões brasileiras, tampouco solucionou a escassez de leitos que precede a pandemia. A ocorrência de filas de pacientes à espera de leitos, derivada tanto da insuficiência de leitos públicos quanto da incapacidade de resposta governamental a termo, bem como da desarticulação entre os setores público e privado de saúde - incluindo as empresas de planos de saúde e os hospitais privados -, sugere a incúria e o insucesso no planejamento das ações para enfrentamento da pandemia. As mudanças de Ministros da Saúde por discordâncias na condução da pandemia e os indícios de fraudes na aquisição de recursos em algumas cidades do país foram agravantes nesse cenário e turbinaram a crise política.

A omissão de informações sobre a existência de filas e a ausência de informações precisas sobre a expansão de leitos Covid-19 no setor privado inviabilizam sobremaneira o planejamento das ações, a realocação de recursos e a formulação de políticas públicas que visem apontar alternativas para o enfrentamento da pandemia.

A organização da rede assistencial insuficiente, tardia e decalcada na estrutura desigual da oferta conjugada com flexibilização precoce do isolamento social agravaram a transmissão e a letalidade da doença no País. Tenta-se difundir, frequentemente com base na ocupação de leitos, uma falsa impressão de normalidade, gerando uma indiferença como se as 'vidas não importassem'. Impõe-se uma interpretação inadequada de resiliência, como se fosse plausível considerar resiliente a aceitação de que critérios econômicos se sobreponham à vida de milhares de pessoas.

Construir hospitais de campanha foi a principal política para ampliar a capacidade instalada e o acesso da população. Países como China, Espanha e Itália também optaram pela organização de hospitais de campanha para aumentar a quantidade de leitos, no entanto, nesses países, a implementação foi realizada de forma célere. No Brasil, onde a oferta de leitos privados, especialmente de terapia intensiva, é sabidamente maior do que a pública, a estratégia priorizada não foi a do uso de recursos existentes. Mesmo diante dos problemas na compra de respiradores e contratação de profissionais, que inviabilizou a abertura de hospitais de campanha ou seu pleno funcionamento, a captação de leitos de hospitais privados já equipados e prontos para receber pacientes em filas à espera de atendimento especializado ficou restrita a determinadas cidades.

Embora experiências internacionais e nacionais demonstrem a viabilidade da medida, em todas as cidades que registraram colapso do sistema de saúde, a opção foi privilegiar a esfera privada em detrimento da vida, uma omissão que vai entrar para este capítulo da nossa história.

O art. $6^{\circ}$ da Constituição Federal de 1988 prevê que a saúde é um direito social, inderrogável, irrenunciável e indisponível. Dessa forma, não pode ser extinto pela lei nem renunciado pela própria pessoa ${ }^{60}$. Diante dessa máxima, pode o poder público se eximir da responsabilidade na prestação de assistência à saúde e acesso aos serviços?

Recorrer ao Poder Judiciário tem sido a via escolhida para a garantia de direitos não atendidos pelos setores público e privado de saúde, e essa prática continua em evidência 
neste momento de crise sanitária, importando destacar a atuação do Poder Judiciário e do Ministério Público na proteção de direitos durante a pandemia. Todavia, o acesso a leitos deveria ser igualitário para todos os indivíduos como diretriz nacional, não sendo necessário pleitear por via judicial um direito constitucional, principalmente em uma situação de urgência na qual é premente a garantia de acesso oportuno.

$\mathrm{O}$ enfrentamento da pandemia poderia promover o fortalecimento e a ampliação do sistema público e do complexo médico-industrial da saúde, diante do aumento dos investimentos públicos, abertura de novos leitos, reconhecimento e valorização do trabalho dos profissionais de saúde, investimentos em ciência e tecnologia e na indústria nacional e atendimento igualitário. Ao contrário, a resposta brasileira à Covid-19, repleta de falhas, acentua fragilidades do poder público e conota renúncia a respeitar a garantia à saúde como um direito social previsto na Constituição Federal.

Enquanto muitos cidadãos brasileiros não conseguirem respirar por inclemência de autoridades governamentais e empresários privados, os princípios de universalidade do SUS ficam questionados; a garantia da vida e dos direitos sociais permanecem em último plano.

A taxa de ocupação de leitos utilizada como principal critério para a flexibilização do isolamento social desfigura a realidade. A imposição de uma suposta volta da normalidade tem sido acompanhada por sugestões de sustar políticas sociais, como auxílios de renda, retração de medidas preventivas e ênfase na recomendação de estratégias farmacológicas sem evidências científicas.
Ainda assim, o principal esforço do governo federal na luta contra o vírus, centrado em drogas antimaláricas e vermífugos, é questionável. Enquanto sobram medicamentos sem comprovação científica, faltam anestésicos para entubar os pacientes em estado crítico.

As desigualdades no acesso aos serviços públicos e privados no País foram blindadas, as estruturas que nutrem as disparidades assistenciais estão vigentes. Tanto a lógica subjacente à Emenda Constitucional no 95/2016 quanto a capacidade de vocalização de interesses e veto a políticas universais de grupos econômicos continuam intactas. Entretanto, detecta-se a afirmação de valores sociais solidários e amplo reconhecimento da relevância do SUS. Uma contradição explícita entre as expectativas da população e as políticas governamentais, que, certamente, acresce tensão à crise e ao debate sobre a reforma do sistema de saúde no Brasil.

\section{Colaboradores}

Costa DCAR (0000-0001-9691-9748)* e Bahia L (0000-0001-8730-2244)* contribuíram para a elaboração do artigo com as seguintes atividades: concepção, planejamento, análise e interpretação dos dados; revisão crítica do conteúdo; e aprovação da versão final do manuscrito. Carvalho EMCL (0000-00029517-7161)*, Cardoso AM (0000-0001-96585899)*e Souza PMS (0000-0002-7058-8386)* contribuíram com as seguintes atividades: revisão crítica do conteúdo e aprovação da versão final do manuscrito. 


\section{Referências}

1. Brasil. Ministério da Saúde. Departamento de Informática do Sistema Único de Saúde (Datasus) [internet]. [Brasília, DF]: Informações de saúde. Rede Assistencial; 2020 [acesso em 2020 jun 21]. Disponível em: http://tabnet.datasus.gov.br/cgi/deftohtm. exe?cnes/cnv/leiutibr.def.

2. Ferguson NM, Laydon D, Nedjati-Gilani G, et al. Report 9: Impact of non-pharmaceutical interventions (NPIs) to reduce COVID-19 mortality and healthcare demand. London: Imperial College London; 2020.

3. Leung K, Wu JT, Liu D, et al. First-wave COVID-19 transmissibility and severity in China outside $\mathrm{Hu}-$ bei after control measures, and second-wave scenario planning. Lancet [internet]. 2020 [acesso 2020 jul 10]; (395):1382-93. Disponível em: https://www. thelancet.com/pdfs/journals/lancet/PIIS01406736(20)30746-7.pdf.

4. World Health Organization. Considerations in adjusting public health and social measures in the context of COVID-19: interim guidance. Geneva: WHO; 2020.

5. Fundação Oswaldo Cruz. Monitora Covid-19. Tendências atuais da pandemia de Covid-19: Interiorização e aceleração da transmissão em alguns estados. Nota Técnica: 2020. Rio de Janeiro: Fiocruz; 2020.

6. Rio de Janeiro (cidade). Prefeitura. Programa Rio de Novo [internet]. [acesso em 2020 jul 13]. Disponível em: https://riocontraocorona.rio/plano-de-retomada/\#: :text=Plano\%20de\%20Retomada\%20 (atualizado\%2010,do\%20dia\%2002\%2F06).

7. Estado de São Paulo. Plano São Paulo [internet]. [acesso em 2020 jul 13]. Disponível em: https://www.saopaulo.sp.gov.br/wp-content/uploads/2020/07/balanco-plano-sp-10072020.pdf.

8. Brasil. Ministério da Saúde. Portaria $\mathrm{n}^{\circ} 414$, de 18 de março de 2020. Autoriza a habilitação de leitos de Unidade de Terapia Intensiva para atendimento exclusivo dos pacientes COVID-19. Diário Oficial da União. 9 Abr 2020.
9. Brasil. Ministério da Saúde, Secretaria de Atenção Especializada à Saúde. Portaria no 237 , de 18 de março de 2020. Inclui leitos e procedimentos na Tabela SUS para atendimento exclusivo dos pacientes COVID-19. Diário Oficial da União. 9 Abr 2020.

10. Brasil. Ministério da Saúde, Departamento de Informática do Sistema Único de Saúde (Datasus) [internet]. 2020. [acesso em 2020 jul 11]. Disponível em: https://wiki.saude.gov.br/cnes/index.php/ Orienta\%C3\%A7\%C3\%B5es_CNES_-_COVID-19.

11. Brasil. Ministério da Saúde, Departamento de Informática do Sistema Único de Saúde (Datasus) [internet]. 2020. [acesso em 2020 jul 11]. Disponível em: https://wiki.saude.gov.br/cnes/index.php/Principais_Conceitos\#: :text=Leitos\%20n\%C3\%A3o\%20 SUS\%3A\%20um\%20leito,em\%20hospitais\%20 privados)\%3B\%20ou.

12. Agência Nacional de Saúde Suplementar. Coronavírus: informe sobre demandas dos beneficiários [internet]. 2020. [acesso em 2020 jun 26]. Disponível em: https://app.powerbi.com/view?r=eyJrIjoiNTMzYjNmZDQtODczOC00ZTFmLWJhNzUtNjdlM2FkMjZ jMGJmIiwidCI6IjlkYmE0ODBjLTRmYTctNDJmNCliYmEzLTBmYjEzNzVmYmU1ZiJ9.

13. Rio de Janeiro (estado). Tribunal de Justiça do Estado do Rio de Janeiro. Boletim Especial COVID-19 (coronavírus) [internet]. 2020. [acesso em 2020 jul 13]. Disponível em: http://conhecimento.tjrj.jus.br/ web/portal-conhecimento/boletimcovid-19_tjrj.

14. São Paulo (estado). Tribunal de Justiça do Estado de São Paulo. Boletim 1 - Grupo de Apoio ao Direito Privado COVID-19 [internet]. 2020. [acesso em 2020 jul 13]. Disponível em: http://www.tjsp.jus.br/Download/Portal/Coronavirus/material/BoletimGapriCovid19.pdf.

15. São Paulo (estado). Tribunal de Justiça do Estado de São Paulo. Boletim 2 - Grupo de Apoio ao Direito Privado COVID-19 [internet]. 2020. [acesso em 2020 jul 13]. Disponível em: http://www.tjsp.jus.br/Downlo- 
ad/Portal/Coronavirus/material/Boletim-Covid-Gapri-02.pdf?637273759985579068.

16. World Health Organization. WHO Director-General's statement on IHR Emergency Committee on Novel Coronavirus (2019-nCoV). Geneva: WHO; 2020.

17. Brasil. Ministério da Saúde. [internet]. [Brasília, DF]: Painel Coronavírus; 2020 [acesso em 2020 jun 23]. Disponível em: https://COVID.saude.gov.br/.

18. Amazonas. Governo do Estado, Fundação de Vigilância em Saúde do Amazonas. Manaus registra menor taxa de ocupação de leitos desde o início da pandemia, informa Susam [internet]. 2020. [acesso em 2020 jul 12]. Disponível em: http://www.fvs.am.gov. br/noticias_view/3964.

19. Ceará. Governo do Estado, Secretaria de Saúde. Boletim epidemiológico [internet]. 2020. [acesso em 2020 jul 12]. Disponível em: https://www.saude.ce.gov.br/ wp-content/uploads/sites/9/2020/02/BOLETIM_ COVID_26_05_2020.pdf.

20. Pernambuco. Governo do Estado, Secretaria de Saúde. COVID-19 em dados [internet]. 2020. [acesso em 2020 jul 12]. Disponível em: https://dados.seplag.pe.gov.br/ apps/corona.html\#regula\%C3\%A7\%C3\%A3o-srag.

21. G1 Pará. Todas as UTIs públicas da rede municipal de Belém estão ocupadas, alerta Sesma. [internet]. G1. 2020 Abr 4 [acesso em 2020 jul 13]. Disponível em: https://gl.globo.com/pa/para/noticia/2020/04/20/ todas-as-utis-publicas-de-belem-estao-ocupadas-alerta-sesma.ghtml.

22. Correia B. Fila de espera de pacientes com COVID-19 aumenta no Rio; hospitais têm leitos, mas faltam profissionais [internet]. G1. 2020 Maio 27. [acesso em 2020 jun 22]. Disponível em: https://gl.globo.com/ rj/rio-de-janeiro/noticia/2020/05/27/fila-de-espera-de-pacientes-com-covid-19-aumenta-no-rio-hospitais-tem-leitos-mas-faltam-profissionais.ghtml.

23. Cerqueira S, Thomas JA, Bruno C. COVID-19: o drama nos hospitais públicos e as saídas para mitigar a crise [internet]. Veja. 2020 Maio 15. [acesso em 2020 jun 22]. Disponível em: https://veja.abril.com.br/saude/COVID-19-o-drama-nos-hospitais-publicos-e-as-saidas-para-mitigar-a-crise/.

24. Agência Nacional de Saúde Suplementar. Boletim COVID-19: saúde suplementar [internet]. 2020. [acesso em 2020 jun 22]. Disponível em: http://www.ans.gov. br/images/Boletim_COVID-19_ANS_Junho_Link.pdf.

25. Alves R, Barreira G. Hospitais privados do RJ têm queda na ocupação de Covid, mas médicos alertam para risco de novo aumento de contágio [internet]. G1. 2020 Maio 5. [acesso em 2020 jul 13]. Disponível em: https://gl.globo.com/rj/rio-de-janeiro/noticia/2020/05/29/hospitais-privados-do-rj-tem-queda-na-ocupacao-de-covid-mas-medicos-alertam-para-risco-de-nova-onda-de-contagio. ghtml.

26. Agência Nacional de Saúde Suplementar. Sala de situação [internet]. 2020. [acesso em 2020 set 16]. Disponível em: https://www.ans.gov.br/perfil-do-setor/ dados-e-indicadores-do-setor/sala-de-situacao.

27. São Paulo (cidade). Câmara Municipal. Coronavírus: fechamento do hospital de campanha do Pacaembu. [internet]. 2020. [acesso 2020 maio 18]. Disponível em: http://www.saopaulo.sp.leg.br/coronavirus/blog/ coronavirus-fechamento-do-hospital-de-campanha-do-pacaembu/.

28. Garret Jr. G. Manaus fecha hospital de campanha após queda de casos da covid-19 [internet]. Exame. 2020 Jun 24. [acesso em 2020 jul 10]. Disponível em: https://exame.com/brasil/manaus-fecha-hospital-de-campanha-apos-queda-em-casos-diarios-da-covid-19/.

29. Castro CS, Holzgrefe Júnior JV, Reis RB, et al. Pandemia da COVID-19: cenário do sistema de saúde brasileiro para o enfrentamento da crise. Res. Soc. Develop. [internet]. 2020 [acesso em 2020 jul 15]; 9(7):e516974383. Disponível em: https://www.arca. fiocruz.br/handle/icict/41585.

30. Portela MC, Pereira CCA, Lima SML, et al. Limites e possibilidades dos municípios brasileiros para o en- 
frentamento dos casos graves de COVID-19 [internet]. 2020. [acesso em 2020 jun 26]. Disponível em: https:// www.arca.fiocruz.br/bitstream/icict/40749/2/LimitesPossibilidadesMunicipiosCOVID-19.pdf.

31. Fihn SD. COVID-19 - Back to the Future. JAMA Intern Med.[internet]. 2020 [acesso em 2020 jul 10]; 180(9):1149-1150. Disponível em: https://jamanetwork.com/journals/jamainternalmedicine/fullarticle/2768360.

32. Ayres F, Lopes H, Yeung L, et al. Judicialização da Saúde no Brasil: perfil das demandas, causas e propostas de solução. Brasília, DF: Instituto de Ensino e Pesquisa; 2019.

33. Alves DC, Bahia L, Barroso AF. O papel da Justiça nos planos e seguros de saúde no Brasil. Cad. Saúde Pública [internet]. 2009 [acesso em 2020 jun 26]; 25(2):279-290. Disponível em: https://www. scielo.br/scielo.php?script=sci_arttext\&pid=S0102-311X2009000200006.

34. Scheffer M. Coberturas assistenciais negadas pelos planos e seguros de saúde em ações julgadas pelo tribunal de justiça do estado de São Paulo. Rev. de Direito Sanitário. 2013; 14(1):122-132.

35. Kozan JF. Por que pacientes com câncer vão à Justiça? Um estudo sobre ações judiciais movidas contra o Sistema Único de Saúde (SUS) e contra os planos de saúde na Cidade de São Paulo. [dissertação]. [São Paulo]: Faculdade de Medicina da Universidade de São Paulo; 2019. 105 p.

36. Vidas iguais. Leitos para todos. Rede solidária em defesa da vida Pernambuco [internet]. 2020. [acesso em 2020 jun 28]. Disponível em: https://www.vidasiguais.com.br.

37. Associação Brasileira de Saúde Coletiva. Campanhas Vidas Iguais e Leitos para Todos unificam ações e inspiram demais redes [internet]. 2020. [acesso em 2020 jun 28]. Disponível em: https://www.abrasco.org.br/ site/noticias/movimentos-sociais/campanhas-vidas-iguais-e-leitos-para-todos-unificam-acoes-e-inspiram-demais-redes/47115/.
38. Grupo de Estudos sobre Planos de Saúde. Grupo de Pesquisa e Documentação sobre o Empresariamento da Saúde (GPDES). As empresas de planos de saúde no contexto da pandemia do coronavírus: entre a omissão e oportunismo. [internet]. 2020. [acesso em 2020 jun 26]. Disponível em: https://sites.usp.br/ geps/planos-de-saude-na-pandemia-do-coronavirus-entre-a-omissao-e-o-oportunismo/.

39. Bahia L. O Brasil deveria criar uma regulação única de leitos públicos e privados no combate ao coronavírus? SIM [internet]. Folha de S. Paulo. 2020 abr 18. [acesso em 2020 jul 13]. Disponível em: https:// wwwl.folha.uol.com.br/opiniao/2020/04/o-brasil-deveria-criar-uma-regulacao-unica-de-leitos-publicos-e-privados-no-combate-ao-coronavirus-sim. shtml.

40. Folha de S. Paulo. Pesquisadores defendem fila única de leitos públicos e privados contra coronavírus [internet]. 2020 Abr 1. [acesso em 2020 jul 19]. Disponível em: https://wwwl.folha.uol.com.br/cotidiano/2020/04/pesquisadores-defendem-fila-unica-de-leitos-publicos-e-privados-contra-coronavirus. shtml.

41. Brasil. Senado Federal. Senado aprova uso de leitos de UTI particulares por pacientes do SUS [internet]. Agência Senado. 2020 Maio 26. [acesso em 2020 jun 24]. Disponível em: https://www12.senado.leg.br/noticias/materias/2020/05/26/senado-aprova-uso-de-leitos-de-uti-particulares-por-pacientes-do-sus.

42. Brasil. Lei $\mathrm{n}^{\circ} 13.979$, de 6 de fevereiro de 2020. Dispõe sobre as medidas para enfrentamento da emergência de saúde pública de importância internacional decorrente do coronavírus responsável pelo surto de 2019. Diário Oficial da União. 7 Fev 2020.

43. Conselho Nacional de Saúde. Recomendação n ${ }^{\circ} 026$, de 22 de abril de 2020. Recomenda aos gestores do SUS que requisitem leitos privados, quando necessário, e procedam à sua regulação única a fim de garantir atendimento igualitário durante a pandemia [internet]. [acesso em 2020 maio 5]. Disponível em: http:// conselho.saude.gov.br/recomendacoes-cns/1131-recomendacao-n-026-de-22-de-abril-de-2020. 
44. Agência Nacional de Saúde Suplementar. Voto ${ }^{\circ}$ 11/2020/DIGES [internet]. 2020. [acesso em 2020 jun 10]. Disponível em: http://www.ans.gov.br/sdcol/ anexo/76499__Voto\%2011.pdf.

45. Monteiro BF. COVID-19: soluções técnicas para ampliar o número de leitos [internet]. Estadão. $2020 \mathrm{Abr}$ 4. [acesso em 2020 maio 19]. Disponível em: https:// politica.estadao.com.br/blogs/fausto-macedo/covid-19-solucoes-tecnicas-para-ampliar-o-numero-de-leitos/.

46. Confederação Nacional do Comércio de Bens, Serviços e Turismo. Gestão unificada de leitos de UTI é desaprovada por seguradoras [internet]. 2020. [acesso em 2020 jun 28]. Disponível em: http://cnc.org.br/ editorias/sistema-comercio/noticias/gestao-unificada-de-leitos-de-uti-e-desaprovada-por-seguradoras.

47. Federação Nacional de Saúde Suplementar [internet]. Presidente da FenaSaúde condena gestão unificada de leitos [internet]. 2020. [acesso em 2020 jun 28]. Disponível em: http://fenasaude.org.br/noticias/ presidente-da-fenasaude-condena-gestao-unificada-de-leitos.html.

48. Nabuco F. Em vez de leitos improvisados em estádios, por que não os contratar na rede privada? Rev. Visão Hospitalar [internet]. 2020 [acesso em 2020 jul 7]; 9(31):10-11. Disponível em: http://fbh.com.br/ wp-content/uploads/2020/04/RevistaHospitalar_ Ed31_PT_27042020_web.pdf.

49. Neves H. O Brasil deveria criar uma regulação única de leitos públicos e privados no combate ao coronavírus? NÃO [internet]. Folha de S. Paulo. $2020 \mathrm{Abr}$ 18. [acesso em 2020 jul 13]. Disponível em: https:// wwwl.folha.uol.com.br/opiniao/2020/04/o-brasil-deveria-criar-uma-regulacao-unica-de-leitos-publicos-e-privados-no-combate-ao-coronavirus-nao. shtml.

50. Koike B. Rio de Janeiro: Fila única de pacientes gera polêmica [internet]. Blog Abramge. 2020 Abr 30. [acesso em 2020 jun 28]. Disponível em: https://blog. abramge.com.br/saude-suplementar/fila-unica-de-pacientes-gera-polemica/.
51. Grupo de Estudos sobre Planos de Saúde. Grupo de Pesquisa e Documentação sobre o Empresariamento da Saúde. Documento da ANS contém erros: questionamentos sobre a Nota Técnica n ${ }^{\circ}$ 4/2020/DIDES da Agência Nacional de Saúde Suplementar (ANS), contrária ao uso de leitos privados por usuários do Sistema Único de Saúde (SUS) durante a pandemia de COVID-19 [internet]. São Paulo: USP; Rio de Janeiro: UFRJ; 2020. [acesso em 2020 jun 26]. Disponível em: https://sites.usp.br/geps/covid-19-documento-da-ans-contem-erros/.

52. Grupo de Estudos sobre Planos de Saúde. Grupo de Pesquisa e Documentação sobre o Empresariamento da Saúde. Crise sanitária e recursos assistenciais para o enfrentamento da COVID-19: notas preliminares sobre orçamentos, leitos, respiradores, testes e equipamentos de proteção individual. Nota Técnica. São Paulo: USP; Rio de Janeiro: UFRJ. No prelo 2020 Set.

53. Conselho Nacional de Secretários de Saúde. Conass informa $\mathrm{n}^{\circ} 230 / 2020$. Nota Técnica CNJ n ${ }^{\circ} 24$ [internet]. 2020. [acesso em 2020 jun 28]. Disponível em: https://www.conass.org.br/conass-informa-n-230-2020-nota-tecnica-cnj-n-24-sobre-adocao-de-medidas-de-gestao-voltadas-a-prevencao-da-judicializacao-da-saude-durante-a-pandemia-da-COVID-19/.

54. Rio Grande do Sul. Governo do Estado. Edital de Chamamento Público nº 014/2020 [internet]. 2020. [acesso em 2020 jun 12]. Disponível em: https://saude.rs.gov.br/upload/arquivos/202005/15172510-edital-contrato-de-leitos-privados.pdf.

55. São Paulo (estado). Decreto $\mathrm{n}^{\circ} 59.396$, de 5 de maio de 2020. Regulamenta a Lei $n^{\circ} 17.340$, de 30 de abril de 2020, que dispõe sobre medidas de proteção da saúde pública e de assistência social e outras medidas para o enfrentamento da COVID-19 [internet]. 2020. [acesso em 2020 maio 15]. Disponível em: http:// diariooficial.imprensaoficial.com.br/nav_v5/index. asp?c=1\&e=20200506\&p=1.

56. Nova Friburgo. Decreto $n^{\circ} 605$, de 08 de junho de 2020. Regulamenta no âmbito do município de Nova Friburgo, a requisição administrativa prevista no art. 
$5^{\circ}$, XXV, da Constituição Federal e dá outras providências [internet]. Diário Oficial Eletrônico do Município de Nova Friburgo. 9 Jun 2020. [acesso em 2020 jun 15]. Disponível em: https://plenussistemas. dioenet.com.br/public/uploads/diarios/2020/06/ bb88b7b0b68214474b4805544e81863a.pdf.

57. Maranhão. Governo do Estado. Governo do Maranhão publica decreto sobre requisição administrativa de leitos privados [internet]. 2020. [acesso em 2020 maio 15]. Disponível em: https://www.ma.gov. br/agenciadenoticias/?p=276405.

58. Brasil. Governo do Distrito Federal. Acordo entre Saúde e rede privada gera 35 leitos de UTI durante a pandemia [internet]. 2020. [acesso em 2020 jul 15]. Disponível em: https://www.agenciabrasilia.df.gov. br/2020/07/09/acordo-entre-saude-e-rede-privada-gera-35-leitos-de-uti-durante-a-pandemia/.

59. Associação Brasileira de Captadores de Recursos. Monitor das doações: COVID-19 [internet]. 2020. [acesso em 2020 jul 10]. Disponível em: https://www.monitordasdoacoes.org.br/.

60. Brasil. Constituição, 1988. Constituição da República Federativa do Brasil. Brasília, DF: Senado Federal; 1988.

Recebido em 20/07/2020

Aprovado em 19/09/2020

Conflito de interesses: inexistente

Suporte financeiro: Conselho de Desenvolvimento Científico

e Tecnológico (CNPq) - Chamada Universal MCTI/CNPq №

01/2016 - Processo no 432128/2016-6 